\title{
Gadofluorine M-enhanced MRI shows involvement of circumventricular organs in neuroinflammation
}

\author{
Eva Wuerfel ${ }^{1,2^{*}}$, Carmen Infante-Duarte ${ }^{1}$, Robert Glumm ${ }^{1}$, Jens T Wuerfel ${ }^{1,3}$
}

\begin{abstract}
Background: Circumventricular organs (CVO) are cerebral areas with incomplete endothelial blood-brain barrier (BBB) and therefore regarded as "gates to the brain". During inflammation, they may exert an active role in determining immune cell recruitment into the brain.

Methods: In a longitudinal study we investigated in vivo alterations of CVO during neuroinflammation, applying Gadofluorine M- (Gf) enhanced magnetic resonance imaging (MRI) in experimental autoimmune encephalomyelitis, an animal model of multiple sclerosis. SJL/J mice were monitored by Gadopentate dimeglumine- (Gd-DTPA) and Gf-enhanced MRI after adoptive transfer of proteolipid-protein-specific T cells. Mean Gf intensity ratios were calculated individually for different CVO and correlated to the clinical disease course. Subsequently, the tissue distribution of fluorescence-labeled Gf as well as the extent of cellular inflammation was assessed in corresponding histological slices.
\end{abstract}

Results: We could show that the Gf signal intensity of the choroid plexus, the subfornicular organ and the area postrema increased significantly during experimental autoimmune encephalomyelitis, correlating with (1) disease severity and (2) the delay of disease onset after immunization. For the choroid plexus, the extent of Gf enhancement served as a diagnostic criterion to distinguish between diseased and healthy control mice with a sensitivity of $89 \%$ and a specificity of $80 \%$. Furthermore, Gf improved the detection of lesions, being particularly sensitive to optic neuritis. In correlated histological slices, Gf initially accumulated in the extracellular matrix surrounding inflammatory foci and was subsequently incorporated by macrophages/microglia.

Conclusion: Gf-enhanced MRI provides a novel highly sensitive technique to study cerebral BBB alterations. We demonstrate for the first time in vivo the involvement of CVO during the development of neuroinflammation.

\section{Background}

The central nervous system (CNS) may no longer be considered immune privileged but rather a site of selective immune activity [1,2]. This so-called restricted immunity is warranted by the barrier function of capillary endothelium, which channels the entry of serum proteins and immune cells from the blood to the CNS or the cerebrospinal fluid (CSF), respectively [1]. Although the blood-brain barrier (BBB) covers most parts of the CNS, certain brain regions including the choroid plexus as well as structures that line the cavity of the third and of the

\footnotetext{
* Correspondence: wuerfel@paedia.ukl.mu-luebeck.de

'Experimental and Clinical Research Center, Charité - University Medicine

Berlin, Charitéplatz 1, 10117 Berlin, Germany

Full list of author information is available at the end of the article
}

fourth ventricle are devoid of a tight $\mathrm{BBB}$ and are in permanent contact to blood-born molecules and cells. These "exposed" areas, called circumventricular organs (CVO), are characterized by a dense capillary network with wide perivascular areas. Assumably, specialized ependymal cells, the tanycytes, act as a flexible barrier controlling the exchange of substances between CVO and the surrounding brain parenchyma as well as the CSF $[3,4]$. Besides neuroendocrine functions, $\mathrm{CVO}$ provide an access route for immune cells into the brain and might therefore guide CNS immune surveillance. The capacity of the choroid plexus to build a bridge for immune cells trafficking from the blood circulation into the CSF and the subarachnoid space was demonstrated in physiological [5] as well as under inflammatory conditions [6]. 
Immune activation was also reported in other $\mathrm{CVO}$ during experimental autoimmune encephalomyelitis (EAE), an animal model of multiple sclerosis (MS), indicating, that these unprotected CNS areas play a key role for pathological immune processes of the brain [7]. However, until recently no reliable method had been available to survey CVO in vivo. Assuming a crucial function as "gates to the brain" for immune cells, the visualization of alterations in $\mathrm{CVO}$ might become of diagnostic and therapeutic value for the assessment of neuroinflammatory conditions, similar to the detection of BBB impairment by gadopentetate dimeglumine- (Gd-DTPA) enhanced MRI, the current gold standard for the evaluation of disease activity in MS. New developments in high field strength MRI and novel contrast media provide optimized means to detect lesions and localize alterations in BBB integrity, even in small rodent disease models such as murine EAE [8]. The novel gadolinium-based contrast agent Gadofluorine M (Gf) was recently shown to facilitate the visualization of CNS lesions and cranial nerve inflammation [8-10]. Gf was originally applied for the detection of malignant lymph nodes [11], atherosclerotic plaques [12], or peripheral nerve damage [13].

In this study, we demonstrated for the first time that Gf-enhanced MRI represents a unique tool to in vivo visualize alterations of CVO. The magnitude of Gf accumulation in the choroid plexus and other CVO increased during active inflammation, correlated with disease activity, and could be used to differentiate between EAE animals and controls. Furthermore, Gf facilitated the detection of otherwise occult inflammatory CNS lesions.

\section{Methods}

\section{Adoptive-transfer EAE}

All experiments were approved by the local animal welfare committee and conformed to the European Communities Council Directive (86/609/EEC). For adoptive transfer EAE, female naïve SJL/J mice were immunized with an emulsion containing $250 \mu \mathrm{g}$ PLP (murine proteolipid peptide p139-151; purity $>95 \%$, Pepceuticals, Leicester, UK) in equal volumes of phosphate buffered saline (PBS) and Complete Freunds Adjuvant (CFA, Difco Laboratories, Detroit, MI, USA) and $4 \mathrm{mg} / \mathrm{ml}$ Mycobacterium tuberculosis H37Ra (Difco Laboratories, Detroit, MI, USA) [14]. Ten days after immunization cells were extracted from draining lymph nodes and restimulated with $12.5 \mu \mathrm{g} \mathrm{PLP} / \mathrm{ml}$ in cell culture medium (RPMI 1640 supplemented with $2 \mathrm{mM} \mathrm{L}$-glutamine, 100 $\mathrm{U} / \mathrm{ml}$ penicillin, $100 \mu \mathrm{g} / \mathrm{ml}$ streptomycin and $10 \%$ fetal calf serum) for 4 days at $37^{\circ} \mathrm{C}$. Then $8-12 \times 10^{6} \mathrm{~T}$-cell blasts in $100 \mu \mathrm{l}$ PBS were injected intraperitoneally into syngenic recipients [15].

Mice were daily weighed and scored for neurological deficits as previously described [16]: 0 , unaffected; 1 , tail weakness or impaired righting on attempt to roll over; 2, paraparesis; 3 , paraplegia; 4, paraplegia with forelimb weakness or complete paralysis; score $>4$, to be sacrificed. Mice with a score of 4 received an intraperitoneal injection of $200 \mu \mathrm{l}$ glucose $5 \%$ daily.

\section{MRI analysis}

After induction of EAE, mice underwent cerebral MRI between day 5 and 16 post $T$ cell transfer on a 7 Tesla rodent scanner (Pharmascan 70/16AS, Bruker BioSpin, Ettlingen, Germany), applying a $20 \mathrm{~mm}$ RF-QuadratureVolume head coil. Animals received anesthesia via facemask with 1.5 - 2.0\% isoflurane (Forene, Abbot, Wiesbaden, Germany) delivered in $100 \% \mathrm{O}_{2}$ under constant ventilation and body temperature control (Bio Trig System, Bruker BioSpin, Ettlingen, Germany).

We acquired axial and coronal T1-weighted images (MSME; TE $10.5 \mathrm{~ms}$, TR $322 \mathrm{~ms}, 0.5 \mathrm{~mm}$ slice thickness, matrix $256 \times 256$, field of view (FOV) $2.8 \mathrm{~cm}$ ) before and after intravenous (i.v.) injection of $0.2 \mathrm{mmol} / \mathrm{kg}$ bodyweight Gd-DTPA (gadopentetate dimeglumine, Magnevist, Bayer-Schering AG, Berlin, Germany), or $0.1 \mathrm{mmol} /$ $\mathrm{kg}$ bodyweight $\mathrm{Gf}$ (Gadofluorine M, kindly provided by Drs. M. Reinhardt and B. Misselwitz, Bayer-Schering AG, Berlin, Germany). Gd-DTPA is the current gold standard for the detection of BBB breakdown in the CNS, showing as hyperintensity on T1-weighted images instantly after i.v. application. With a blood half-life of $20 \mathrm{~min}$ it is largely excreted from the organism after $3 \mathrm{~h} \mathrm{[17].} \mathrm{Gf} \mathrm{is} \mathrm{a}$ modified amphiphilic gadolinium complex with a molecular weight of about $1.53 \mathrm{~g} / \mathrm{mol}$, also generating bright enhancement on T1-weighted images. Gf was designed by adding a perfluoroctyl chain to a gadolinium containing macrocycle. Gf interacts with hydrophobic proteins of the extracellular matrix such as collagen, proteoglycans, decorin and tenascin, and it is largely bound to serum albumin after i.v. administration [18]. It has a plasma half-life of $15.6 \mathrm{~h} \mathrm{[19].} \mathrm{The} \mathrm{dose} \mathrm{applied} \mathrm{in} \mathrm{this}$ study was approved by previous MRI studies $[8,9]$; it is far below the estimated lethal dose of $5 \mathrm{mmol} / \mathrm{kg}$ [11].

We investigated a total number of 28 mice by MRI, comprising $21 \mathrm{EAE}$ and 7 control mice. Fifteen EAE mice initially received Gd-DTPA. If BBB breakdown could be detected, Gf was applied and MRI repeated after $24 \mathrm{~h}$, as established previously [8]. A subgroup of 4 EAE mice was additionally imaged to assess early kinetics $1 \mathrm{~h}$ and $6 \mathrm{~h}$ after Gf injection. Two mice were followed up until complete clinical remission in order to investigate longitudinal Gf signal decay. A further group of $6 \mathrm{EAE}$ mice received $\mathrm{Gf}$ pre-labelled with the red fluorescent marker Cy3.5 (Gf-Cy3.5) for subsequent immunofluorescence histology. Seven naïve mice served as healthy controls. The kinetic of disease was exclusively studied in vivo owing to ethical concerns, which 
forced us to minimize the number of animals investigated. Histological analyses were performed after the final MR acquisitions.

\section{Histology}

Mice were perfused for post mortem analysis in the acute disease phase within 2 to 5 days after onset. Following the final MRI, brain and spinal cord were prepared for histology, as previously described [8]. Every second slice was stained with hematoxylin and eosin (H\&E) to assess inflammation. For the evaluation of Gf distribution, consecutive slices were stained with Hoechst 33258 nuclear stain (1:10000, Molecular Probes, Leiden, the Netherlands) to visualize cellular organization. We performed immunohistochemical staining against IBA-1 to identify macrophages/microglia, using the primary antibody rabbit anti-IBA-1 (1:1000, Wako Chemicals, Neuss, Germany) and goat anti-rabbit Cy2 (Amersham, Muenchen, Germany) as secondary antibody [8]. Selected sections were examined by epifluorescence microscopy and digitally photographed (Olympus BX-51, Hamburg, Germany).

\section{Data analysis}

MRI data were coregistered and corrected for magnetic field inhomogeneity using MIPAV 6.1 (Center for Information Technology, National Institutes of Health, Bethesda, MD, USA). Statistical analysis was performed with GraphPad Prism 4.0c (GraphPad Software, Inc., San Diego, CA, USA). We determined the T1 lesion load of each individual EAE animal by calculating the volume of cranial Gf enhancement in T1-weighted sequences, applying a semi-automated procedure described previously [20]. In contrast to Gf, enhancement after Gd-DTPA administration was diffuse without clearly obtainable borders and furthermore, was often masked by strong intraventricular Gd-DTPA signal due to Gd-DTPA leakage into the CSF. Accordingly, we were not able to reliably quantify the volume of GdDTPA-enhancing lesions. The Gf lesion volume was correlated to 1) the EAE score at the day of imaging and 2) the day of clinical EAE onset after immunization, applying Spearman's nonparametric analysis.

For the assessment of Gf enhancement in CVO, regions of interest (ROI) were placed in corresponding positions in all animals, and the mean ROI signal intensity as well as the standard deviation was calculated. We evaluated the subfornicular organ (SFO), the organum vasculosum of the lamina terminalis (OVLT), the median eminence (ME) and the area postrema (AP) in coronal slices. The most reliable ROI placement of the choroid plexus could be achieved in axial slices of the lateral ventricles. Choroid plexus examination was generally more rater demanding due to its widespread expansion inside the ventricles with high interindividual anatomic shape differences, and was more prone to partial volume contaminations compared to other areas. The mean signal intensitiy of each ROI was expressed as Gf mean intensity ratio by division with the mean signal intensity of a masseter muscle ROI for unbiased and stable comparison between individuals.

Two-tailed Mann-Whitney-U tests were applied to express differences of the Gf mean intensity ratio in CVO between naïve versus EAE animals. Correlation analyses were performed between the Gf mean intensity ratio and 1) the EAE score at the day of MRI and 2) the day of clinical disease onset using Spearman's nonparametric analysis. A receiver-operating characteristic (ROC) was used to analyze the validity of the Gf mean intensity ratio in $\mathrm{CVO}$ as diagnostic test to distinguish between EAE mice and controls.

\section{Results}

Clinical EAE course and contrast-enhancing lesions (CEL)

Transfer of PLP-specific T cells into recipient animals resulted in the development of clinical signs in 18 out of 21 mice within 6-13 (mean 9.6) days after immunization. However, contrast-enhancing lesions (CEL) were detectable in all 21 mice in T1-weighted images after Gd-DTPA and Gf application. Animals progressed to a peak EAE score of 2.6 (1-4) within 2 days after onset, and developed an average Gf-enhanced T1-hyperintense lesion volume of $8.31 \mathrm{~mm}^{3}$ (table 1). The Gf lesion volume correlated significantly with the EAE score at imaging, but not with the day of clinical EAE onset after immunization (table 2).

Inflammatory plaques were widely distributed throughout the brain, with predominance to the brainstem and the periventricular region. In 15 mice receiving both, Gd-DTPA and Gf, a total number of $61 \mathrm{CEL}$ was detected. Among these, 26 were exclusively visible after Gf administration, but not on Gd-DTPA-enhanced MRI. The visualization of optic nerve inflammation was particularly facilitated by Gf application (table 3). Gfenhancing plaques appeared concise with welldelineated borders in comparison to more diffusely Gd-DTPA-enhancing lesions (figure 1).

Gf tissue enhancement evolved with time delay compared to Gd-DTPA, which indicated BBB breakdown instantly after application. After $1 \mathrm{~h}$, Gf CEL were detectable with circumscribed subtle parenchymal signal enhancement, and developed further within $24 \mathrm{~h}$ (figure $2 \mathrm{~A}$, arrowheads). T1-hyperintensity maximum was reached $24 \mathrm{~h}$ after application, followed by a gradual signal drop until complete remission to baseline values after 14 days (figure 2B).

\section{Visualization of CVO alterations by Gf-enhanced MRI}

In addition to inflammatory plaques, Gf revealed alterations in CVO during acute CNS inflammation. Unlike 
Table 1 Clinical EAE course and Gf lesion load

\begin{tabular}{llll}
\hline Mouse & $\begin{array}{l}\text { EAE score at Gf } \\
\text { imaging }\end{array}$ & $\begin{array}{l}\text { Gf lesion load in } \\
\mathbf{m m}^{3}\end{array}$ & $\begin{array}{l}\text { Day of clinical } \\
\text { onset }\end{array}$ \\
\hline 1 & 1 & 30.5933 & 10 \\
2 & 3 & 22.0243 & 9 \\
3 & 3 & 19.7933 & 11 \\
4 & 1 & 17.3736 & 13 \\
5 & 4 & 13.7473 & 6 \\
6 & 2 & 12.2391 & 7 \\
7 & 1 & 10.4559 & 9 \\
8 & 2 & 9.6347 & 9 \\
9 & 1 & 7.4567 & 9 \\
10 & 2 & 6.3080 & 9 \\
11 & 2 & 6.0699 & 9 \\
12 & 2 & 4.8076 & 11 \\
13 & 1 & 3.2377 & 11 \\
14 & 1 & 3.0483 & 11 \\
15 & 1 & 1.8621 & 11 \\
16 & 0 & 1.4209 & 11 \\
17 & 0 & 1.3857 & - \\
18 & 3 & 0.9528 & 8 \\
19 & 1 & 0.8987 & 9 \\
20 & 0 & 0.7135 & - \\
21 & 0 & 0.5301 & - \\
\hline mean & $1.48(+/-1.12)$ & $8.31(+/-8.34)$ & $9.61(+/-1.69)$ \\
$($ SD) & & & \\
\hline Afer & & & \\
\hline
\end{tabular}

After detection of cranial blood-brain barrier breakdown by Gd-DTPA, 0.1 $\mathrm{mmol} / \mathrm{kg}$ bodyweight Gf was applied, and MRI repeated after $24 \mathrm{~h}$. The EAE score at the day of Gf imaging and the corresponding lesion volume in T1weighted Gf-enhanced images are presented. The day of clinical onset is given as days after $\mathrm{T}$ cell transfer.

the delayed accumulation in inflammatory plaques, bright enhancement of the choroid plexus was evident already $1 \mathrm{~h}$ after Gf injection. Applying Gd-DTPA, the evaluation of the choroid plexus was not reliably feasible, since it was frequently obscured by leakage of GdDTPA into the CSF (figure 3).

Gf enhancement of the choroid plexus was a prominent finding in all 21 EAE mice investigated (figure 4, arrow). Gf accumulation was also observed in other CVO including the OVLT (figure 4, ellipse), the SFO (figure 4, square), the ME (figure 4, arrowhead) and the AP (figure 4, circle). Since CVO may represent major entry sites for immune cells into the CNS also under physiological conditions $[6,21,22]$, we were interested to see whether Gf could visualize CVO "gates" in naïve animals, too. In all 7 healthy control mice, subtle Gf enhancement in CVO was detectable (figure $4,3^{\text {rd }} \mathrm{col}-$ umn), although considerably less pronounced compared to EAE mice. We did not assess Gf enhancement in healthy mice longitudinally.

For a quantitative analysis of differences between EAE animals and naïve mice, we calculated the mean signal intensity ratio $24 \mathrm{~h}$ after $\mathrm{Gf}$ application in the CVO within these two groups (figure 5). The Gf mean intensity ratio was significantly higher in EAE mice compared to controls in the choroid plexus $(\mathrm{p}=0.008)$, the SFO $(\mathrm{p}=0.020)$ and the AP $(\mathrm{p}=0.016)$. No difference was detected for OVLT and ME.

\section{Gf enhancement in CVO correlates with disease severity}

Within EAE animals, Gf mean intensity ratios of SFO and AP correlated closely with the EAE score at the day of the MRI investigation; mice with higher disease severity showed significantly brighter Gf enhancement (table 2 and figure 6A and 6B). Additionally, the Gf mean intensity ratio of the SFO correlated inversely with the time span until onset of clinical disease after $\mathrm{T}$ cell transfer; accordingly, Gf enhancement of SFO was significantly brighter in EAE mice with early disease onset (table 2 and figure 6C). The quantitative analysis of the choroid plexus was technically more demanding compared to CVO regions surrounded by brain parenchyma such as SFO and AP, due to its expansion throughout the ventricles with high interindividual anatomical shape differences. Correlations between clinical EAE features and choroid plexus enhancement failed to reach statistical significance, potentially due to partial volume contaminations of CSF when conducting a standardized ROI based semi-automated quantitative analysis.

\section{Gf enhancement of CVO differentiates EAE from control animals}

We were further interested whether the quantitative assessment of Gf enhancement in different CVO could serve as diagnostic tool to distinguish between EAE and

Table 2 Gf enhancement correlates with clinical EAE parameters

\begin{tabular}{|c|c|c|c|c|c|c|}
\hline & \multicolumn{3}{|c|}{ EAE score at Gf imaging } & \multicolumn{3}{|c|}{ Day of clinical onset } \\
\hline & Gf lesion volume & SFO & AP & Gf lesion volume & SFO & AP \\
\hline Spearman $r$ & 0.56 & 0.66 & 0.53 & -0.09 & -0.45 & -0.35 \\
\hline $95 \% \mathrm{Cl}$ & 0.15 to 0.80 & 0.30 to 0.85 & 0.08 to 0.79 & -0.54 to 0.41 & -0.74 to -0.01 & -0.70 to 0.14 \\
\hline P value & 0.009 & 0.001 & 0.021 & 0.732 & 0.043 & 0.147 \\
\hline Significance & $* *$ & $* *$ & * & no & * & no \\
\hline
\end{tabular}

Correlation of clinical EAE parameters to total Gf lesion volume in T1-weighted images and Gf enhancement (mean intensity ratio) in the subfornicular organ (SFO) and the area postrema (AP). Correlations for other circumventricular organs were not statistically significant.

*: $\mathrm{P}$ value $<0.05 ;{ }^{* *}$ : $\mathrm{P}$ value $<0.01 ; \mathrm{Cl}$ : confidence interval. 
Table 3 Contrast-enhancing lesions (CEL) exclusively detected by $\mathbf{G f}$

\begin{tabular}{|c|c|c|c|c|}
\hline Brain region & $\begin{array}{l}\text { Total } \\
\text { number } \\
\text { of CEL }\end{array}$ & $\begin{array}{l}\text { Number of } \\
\text { CEL detected } \\
\text { with Gd- } \\
\text { DTPA and Gf }\end{array}$ & $\begin{array}{l}\text { Number of } \\
\text { CEL } \\
\text { detected } \\
\text { exclusively } \\
\text { with Gf }\end{array}$ & $\begin{array}{l}\text { Percentage } \\
\text { of CEL } \\
\text { detected } \\
\text { exclusively } \\
\text { with Gf }\end{array}$ \\
\hline Brain stem & 14 & 10 & 4 & 28.6 \\
\hline Periventricular & 11 & 9 & 2 & 18.2 \\
\hline Optic nerve & 10 & 1 & 9 & 90 \\
\hline $\begin{array}{l}\text { Trigeminal } \\
\text { nerve }\end{array}$ & 10 & 6 & 4 & 40 \\
\hline Cerebellum & 9 & 6 & 3 & 33.3 \\
\hline $\begin{array}{l}\text { Vestibular } \\
\text { nerve }\end{array}$ & 5 & 2 & 3 & 60 \\
\hline \multirow[t]{2}{*}{ Cortex } & 2 & 1 & 1 & 50 \\
\hline & 61 & 35 & 26 & 42.6 \\
\hline
\end{tabular}

Distribution and number of contrast-enhancing lesions (CEL) after Gd-DTPA and Gf administration in 15 mice investigated with both contrast agents.

healthy mice. ROC curves were calculated separately for the Gf mean intensity ratio of the choroid plexus, the SFO and the AP. The area under the ROC curve was highly significant for the choroid plexus $(\mathrm{p}=0.007)$ as well as for the SFO $(\mathrm{p}=0.018)$ and the AP $(\mathrm{p}=0.014)$. The Gf mean intensity ratio of the choroid plexus was the best discriminator to identify EAE animals with a sensitivity of $89 \%$ and a specificity of $80 \%$ using a cutoff value of 0.9826 (table 4 ).

\section{Gf accumulates in the extracellular matrix and is incorporated by monocytic cells}

In order to investigate cellular or molecular mechanisms of $\mathrm{Gf}$ tissue accumulation, we used carbocyanine dye (Cy3.5) prelabeled contrast agent. MR images were acquired $24 \mathrm{~h}$ and $72 \mathrm{~h}$ post application. Directly after MR measurements, mice were sacrificed and analyzed histologically. Twentyfour hours post injection, Gf-Cy3.5 produced a strong signal in immune fluorescence microscopy, matching the sites of contrast enhancement in MRI (figure 7A and 7B). Fluorescence was most intense within the center of the lesion and vanished towards the periphery (figure 7A3 and 7B3). Gf appeared to enrich homogeneously in the extracellular matrix in foci with high immune cell infiltration (figure 7B4). In corresponding $H \& E$ staining, these areas were identified as inflammatory perivascular cuffs typically seen in EAE (figure 7A2). Lesion sites were often found in vicinity to CVO. Please note the high number of periventricular lesions (table 1) in the proximity to the choroid plexus. Occasionally, the cell formation suggested migration of immune cells from $\mathrm{CVO}$ to inflammatory cuffs (figure 7A2). Gf enrichment was also detected in inflammatory sites located at the meninges (figure 7B). However, this was always associated with a focal inflammatory lesion;

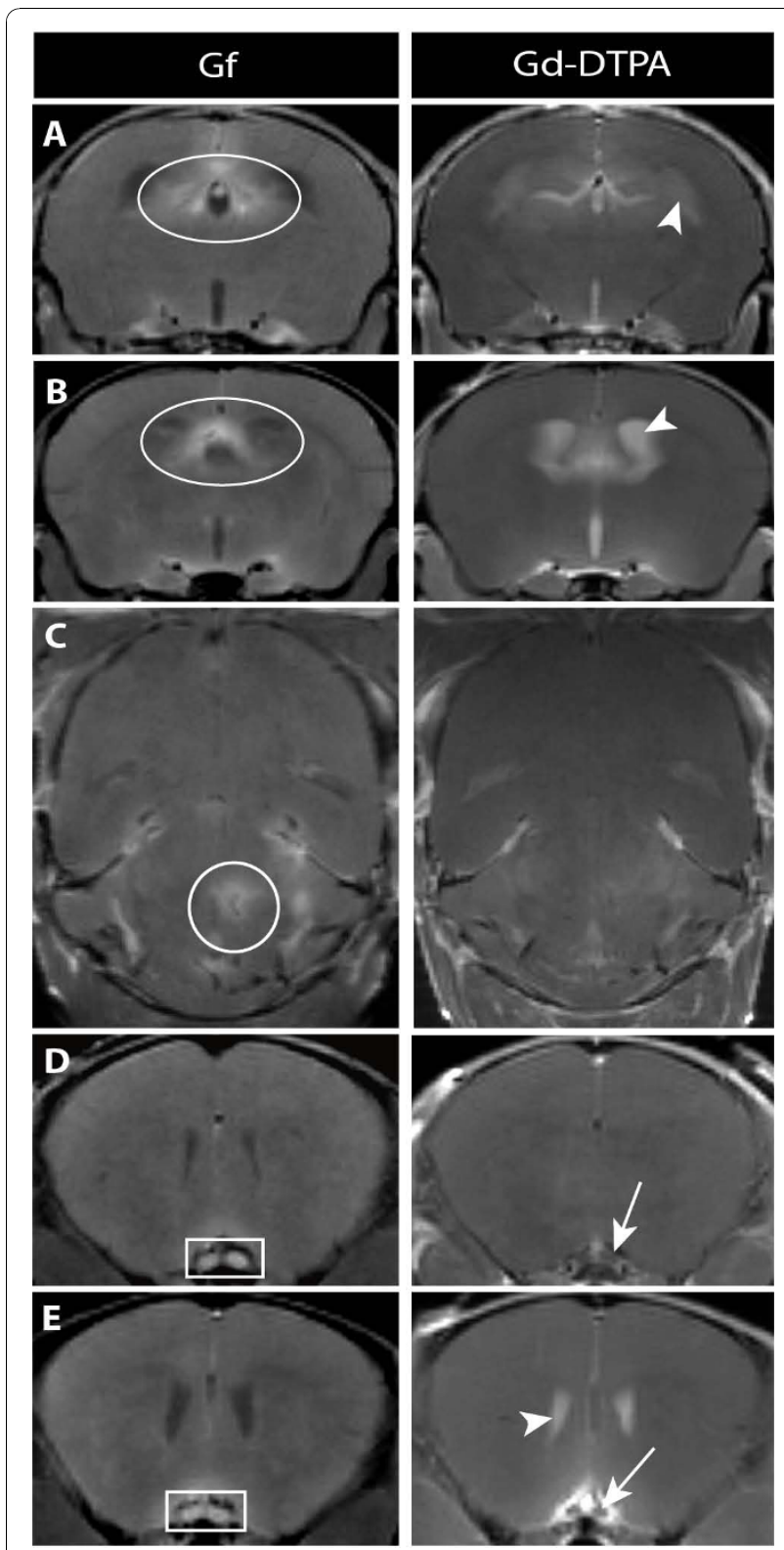

Figure $1 \mathbf{G f}$ facilitates the detection of inflammatory plaques Coronal (A, B, D, E) and axial (C) T1- weighted images are depicted $24 \mathrm{~h}$ after Gf injection (left column) and immediately after Gd-DTPA injection (right column). Periventricular pathology was better assessable applying Gf (A and B: ovoid). The disruption of the blood-CSF barrier frequently caused leakage of contrast agent into the CSF (A, B, E: arrowhead), initially obscuring periventricular lesions. A parenchymal midbrain lesion is shown exemplary (C: encircled) that was not seen with Gd-DTPA. Visualization of optic neuritis ( $D$ and $E$ : square) was markedly improved by Gf, since neighboring intravascular signal prohibited the unambiguous determination on Gd-DTPA-enhanced images ( $D$ and $E$ : arrow).

we did not detect a generalized meningeal Gf enhancement. Although the MR signal did not change significantly within the first $72 \mathrm{~h}$, microscopically Gf formed scattered spots of bright red fluorescence neighbouring 
A
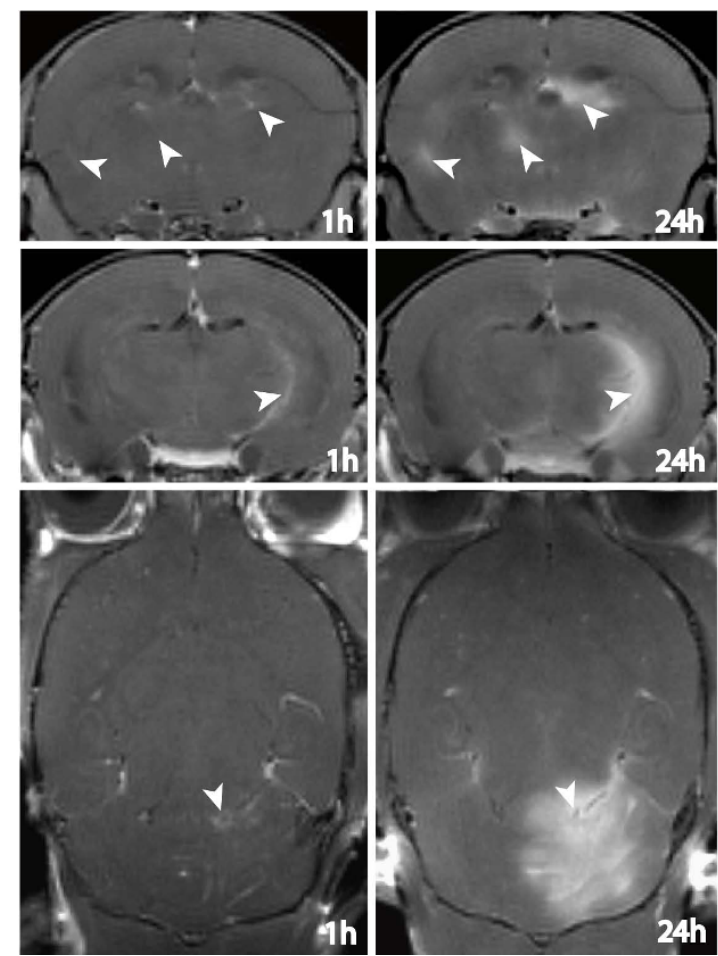

B
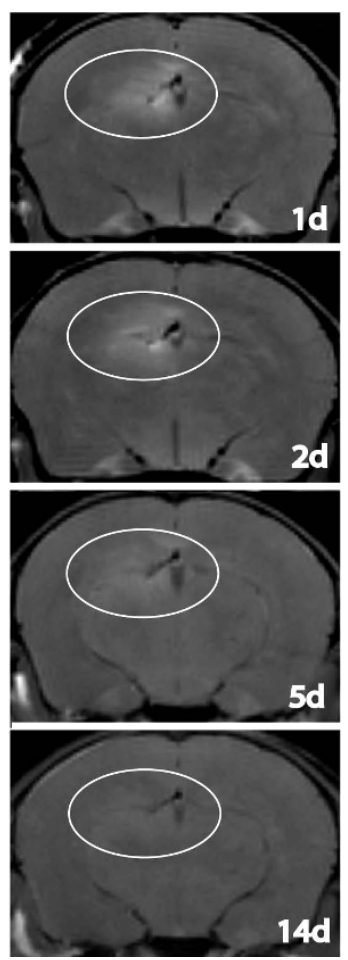

Figure 2 Time course of Gf enhancement. A: Inflammatory plaques located periventricularly (upper row), close to the parahippocampal fissure (middle row), and within the cerebellum (lower row) are depicted to exemplify the minor signal enhancement $1 \mathrm{~h}$ post Gf application compared to the vast parenchymal enrichment $24 \mathrm{~h}$ after Gf injection. B: Gradual Gf signal decline over a time period of 14 days is demonstrated in a periventricular lesion.

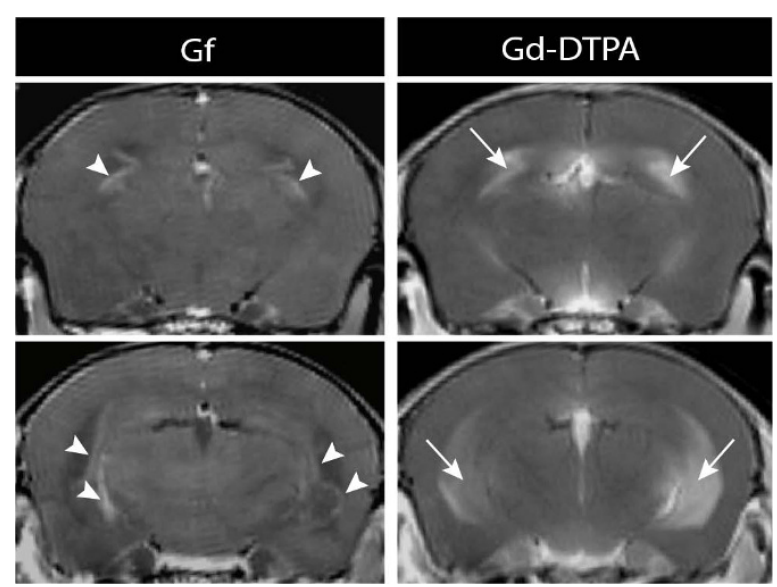

Figure 3 Early Gf enhancement of the choroid plexus. Coronal T1-weighted images are depicted $1 \mathrm{~h}$ after Gf injection (left column) and immediately after Gd-DTPA injection (right column). Applying Gf, distinct enhancement of the choroid plexus (arrow heads) and other CVO was evident early after intravenous injection and allowed for a reliable quantitative signal analysis. In contrast, after injection of Gd-DTPA, the disruption of the blood-CSF barrier frequently caused leakage of contrast agent into the CSF (arrows) obscuring the choroid plexus and periventricular lesions. cell nuclei after initial homogeneous tissue fluorescence, likely due to the accumulation in cytosolic vesicles. Counterstaining with IBA-1 revealed an incorporation of Gf-C3.5 by macrophages/microglia, predominantly in regions with prominent cellular infiltrates detected by H\&E staining. A particularly high density of IBA-1 positive cells with intracellular Gf-C3.5 deposits was detected in the choroid plexus (figure 7C).

\section{Discussion}

This study provides the first in vivo evidence for the participation of the choroid plexus and other CVO in neuroinflammation. Applying the novel MRI contrast agent Gf in high field MRI, we successfully visualized alterations in the $\mathrm{CVO}$, which in part, correlated to the severity of CNS inflammation. Transmigration across the choroid plexus is a well defined entry route for leucocytes into the CNS [1]. Already under physiological conditions, immune cells home to the choroid plexus and other CVO [23]. Macrophages and dendritic cells, key players in CNS antigen presentation, have been localized to the choroid plexus of naïve mice $[6,24]$. Recent histopathological studies stressed the role of the $\mathrm{CVO}$ also during inflammation, suggesting an initial 

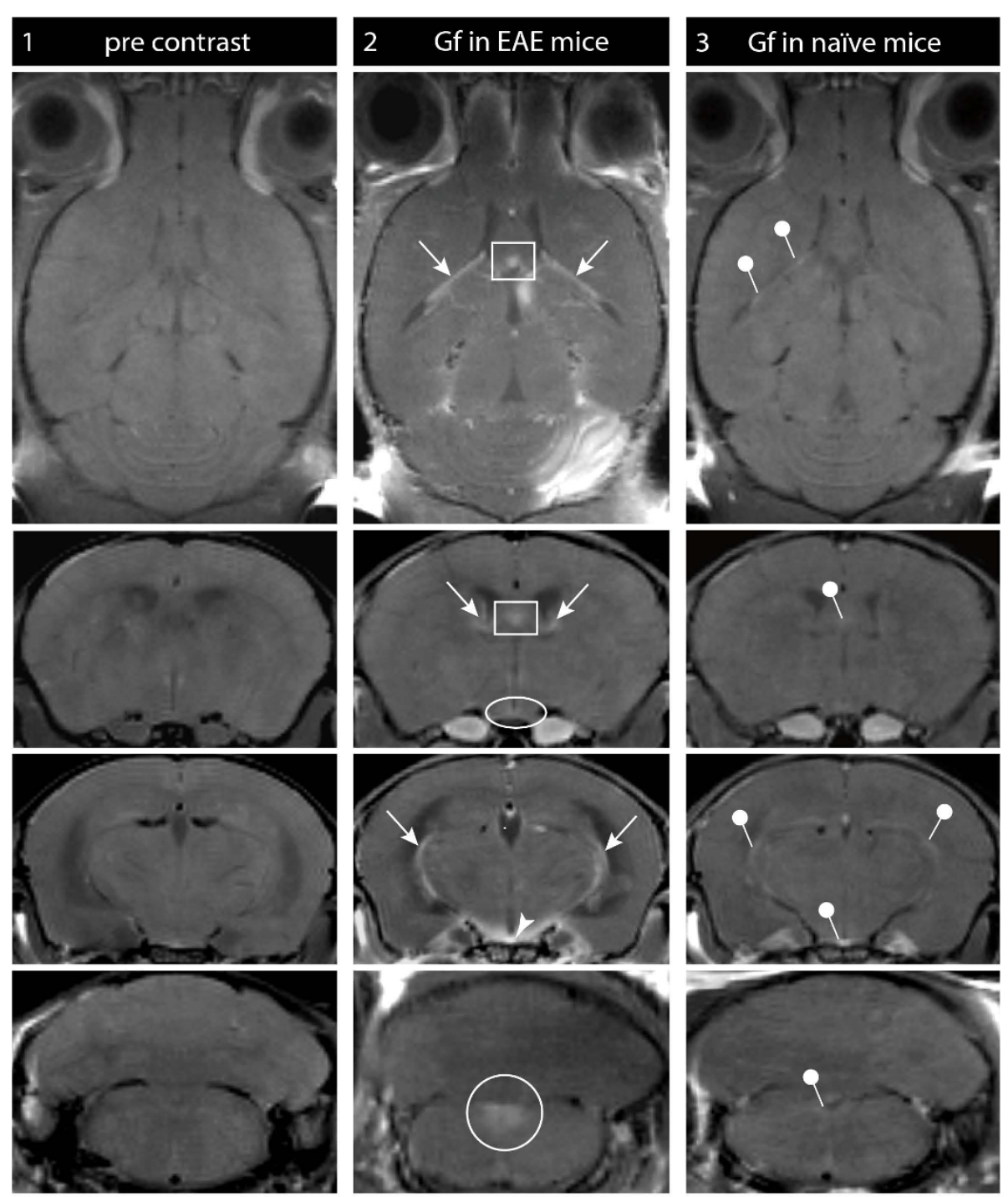

Figure 4 Gf enhancement in circumventricular organs (CVO). Gf distinctly enhanced the choroid plexus and other CVO in naïve control mice and more pronounced after induction of EAE: T1-weighted images of the CVO are shown in axial (row 1) and coronal (row 2-4) orientation. Images were acquired prior to administration of contrast agent (column 1), and $24 \mathrm{~h}$ after Gf application in EAE (column 2) and naïve control mice (column 3). Enhancement of the choroid plexus (arrows), the subfornicular organ (square), the organum vasculosum of the lamina terminalis (ovoid), the median eminence (arrowhead) and the area postrema (circle) are clearly marked in the EAE mice (column 2). In naïve mice, only subtle CVO enhancement was detectable (column 3, pins).

recruitment of encephalitogenic $\mathrm{T}$ cells via the choroid plexus in EAE: Antigen presentation and subsequent reactivation of $\mathrm{T}$ lymphocytes is facilitated due to the presence of macrophages and microglia [25] and the upregulation of major histocompatibility complex antigens in these areas $[7,26]$. Expression of ICAM-1 and VCAM-1, molecules that are crucial for lymphocyte adhesion and transmigration, were induced on choroid plexus epithelium [6] and other CVO during inflammation [7].

Although MRI has emerged as a powerful tool to assess disease activity in MS and EAE, alterations of
CVO during inflammation have not been monitored in vivo up to date. Nevertheless, the invasion of macrophages into inflammatory plaques was demonstrated in vivo in a recent study on MS patients. This finding was partially independent from simultaneous BBB breakdown, and the route of entry is still not completely understood [27]. We hypothesized that Gf-enhanced MRI might help to elucidate kinetics of CNS immune surveillance. Gf was originally developed as a marker for detecting lymph node metastasis in MR lymphography [11]. Meding et al. demonstrated, that Gf binds to serum albumin and components of the extracellular 


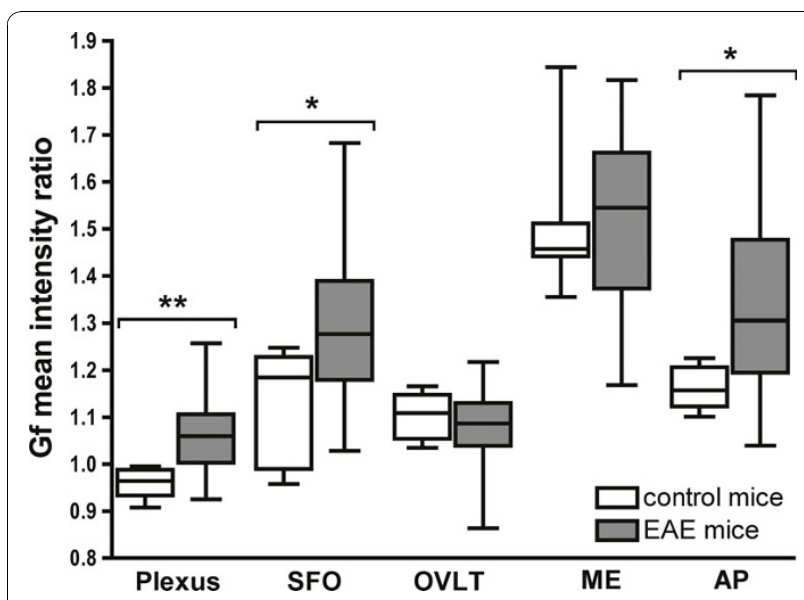

Figure 5 Quantitative comparison of Gf enhancement in circumventricular organs (CVO). Gf enhancement was

quantitatively assessed computing the Gf mean intensity ratio of the CVO in T1-weighted images $24 \mathrm{~h}$ after Gf application. The comparison between EAE and naïve control animals was performed using two-tailed Mann-Whitney-U tests. Gf enhancement in EAE mice was significantly higher in the choroid plexus (**: $P=0.008)$, the subfornicular organ $(*: P=0.020)$ and the area postrema $(*: P=$ 0.016). SFO: subfornicular organ; OVLT: organum vasculosum of the lamina terminalis; ME: median eminence; AP: area postrema. matrix such as collagens, proteoglycans, fibronectin and tenascin [18], explaining a possible mechanism of Gf accumulation in atherosclerotic plaques [12,28]. Furthermore, Gf is taken up by macrophages in vivo and in vitro $[13,29]$ and thus could highlight spots of immune activity in autoimmune neuritis, EAE and peripheral nerve degeneration $[8,9,13,30]$. Recently, Gf was also applied to detect disease progression in an animal model of muscular dystrophy [31].

Here we demonstrate, that Gf accumulates weakly in the choroid plexus and other $\mathrm{CVO}$ involved in gating immune cell migration into the CNS in naive mice, indicating an immunological function of those organs under physiological conditions. Interestingly, after initialization of EAE, pronounced Gf enhancement of the choroid plexus, the SFO and the AP became visible. Whereas leakage of Gd-DTPA into the CSF often prevented a reliable evaluation of the choroid plexus or periventricular brain regions in EAE mice, Gf-enhanced MRI allowed for the detailed quantitative evaluation of signal alterations in these areas. A correlation analysis in EAE mice exposed that high Gf enhancement of the SFO and the AP went along with an early disease onset and severe clinical affection. Regarding the SFO, these
A

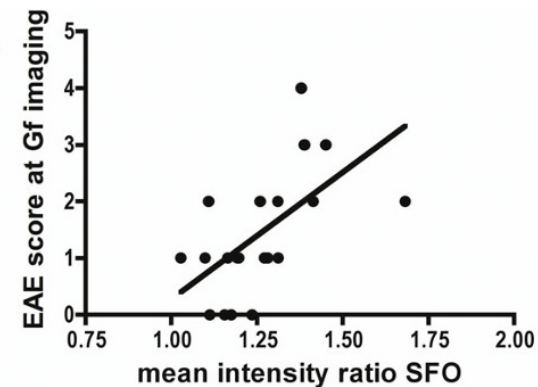

B

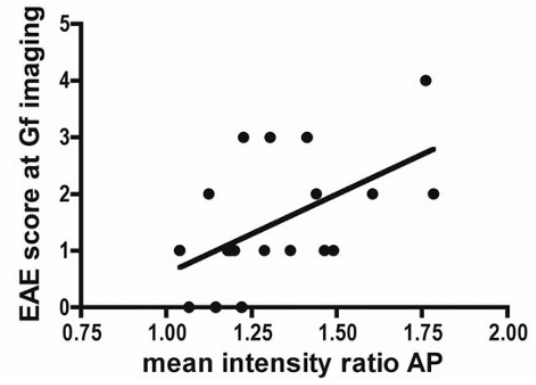

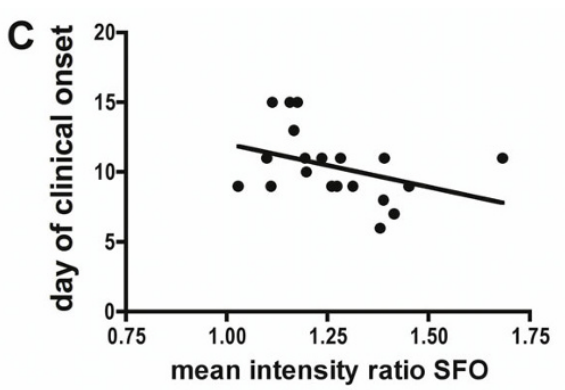

D

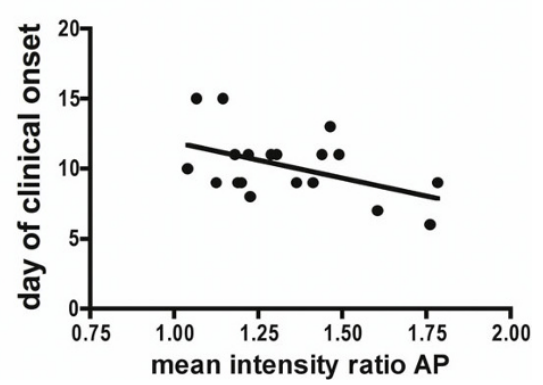

Figure 6 Gf enhancement correlates with clinical parameters. Correlation analyses were performed according to Spearman. The signal intensity (Gf mean intensity ratio) of the subfornicular organ (SFO) $(A, P=0.001)$ and the area postrema $(A P)(B, P=0.021) 24 \mathrm{~h}$ post injection correlated significantly to the corresponding EAE scores at the time of scanning. Additionally, the time needed from $T$ cell transfer to disease onset correlated inversely with the mean intensity ratio of the SFO $(C, P=0.043)$ but not the AP $(D, P=0.147)$. Each dot depicts the data point of one mouse. For further statistics see table 2. 
Table 4 Gf enhancement differentiates between EAE animals and healthy controls

\begin{tabular}{|c|c|c|c|}
\hline & $\begin{array}{l}\text { Choroid } \\
\text { plexus }\end{array}$ & SFO & AP \\
\hline $\begin{array}{l}\text { Area under the ROC } \\
\text { curve }\end{array}$ & 0.90 & 0.81 & 0.84 \\
\hline $\begin{array}{l}\text { Standard error (95\% } \\
\text { Cl) }\end{array}$ & $\begin{array}{l}0.07 \text { (0.77 to } \\
1.03)\end{array}$ & $\begin{array}{l}0.09 \text { (0.64 to } \\
0.98)\end{array}$ & $\begin{array}{l}0.08 \text { (0.68 to } \\
1.01)\end{array}$ \\
\hline P value (two-tailed) & 0.007 & 0.018 & 0.014 \\
\hline Significance & $* *$ & * & * \\
\hline Cutoff MIR & $>0.9826$ & $>1.232$ & $>1.189$ \\
\hline Sensitivity (95\% Cl) & $\begin{array}{l}89 \%(65 \text { to } \\
99 \%)\end{array}$ & $\begin{array}{l}67 \%(41 \text { to } \\
87 \%)\end{array}$ & $\begin{array}{l}82 \%(57 \text { to } \\
96 \%)\end{array}$ \\
\hline Specificity (95\% Cl) & $\begin{array}{l}80 \%(28 \text { to } \\
100 \%)\end{array}$ & $\begin{array}{l}86 \%(42 \text { to } \\
100 \%)\end{array}$ & $\begin{array}{l}83 \%(36 \text { to } \\
100 \%)\end{array}$ \\
\hline Likelihood ratio & 4.44 & 4.67 & 4.94 \\
\hline
\end{tabular}

ROC analysis of the Gf enhancement (mean intensity ratios) in circumventricular organs, differentiating between EAE animals and controls. Exemplary cut-off values and the resulting sensitivity, specificity and likelihood ratio are presented for each region.

ROC: receiver operating characteristic; SFO: subfornicular organ; AP: area postrema; *: $\mathrm{P}$ value $<0.05$; ${ }^{*}: \mathrm{P}$ value $<0.01$; MIR: mean intensity ratio; $\mathrm{Cl}$ : confidence interval.

associations were even higher than the correlation of the same clinical parameters to the Gf lesion volume. Furthermore, we found that calculating the Gf mean intensity ratio in CVO can be applied as diagnostic tool to discriminate EAE from control animals, with a sensitivity of $89 \%$ and a specificity of $80 \%$ for the choroid plexus. In histological sections, we could confirm an accumulation of Gf in the choroid plexus stroma initially and a subsequent internalization into macrophages/ microglia resident in the choroid plexus after $72 \mathrm{~h}$. However, we did not detect significant alterations of the enhancement pattern in OVLT and ME during EAE. The predominance of the observed changes in periventricular and brain stem CVO might reflect a heightened regional vulnerability in developing inflammatory plaques during EAE in these areas [8].

Furthermore, we noted a high sensitivity of Gf to detect BBB leakage and associated parenchymal inflammation, particularly optic neuritis, resulting in a $42 \%$ increased number of contrast-enhancing lesions compared to Gd-DTPA-enhanced MRI, in line with a recent report by Bendszus et al. [9]. In the circulation, Gf is largely bound to albumin [18]. Brain parenchymal enhancement is due to a locally disturbed BBB and subsequent capturing of $\mathrm{Gf}$ molecules by protein interaction [10]. There is no evidence for an active transport mechanism of Gf across the intact BBB. Gf accumulated with delay within CNS lesions yielding a signal intensity peak after $24 \mathrm{~h}$ in contrast to Gd-DTPA that immediately passed the disrupted BBB. Gf enhancement, as quantified in our study, thus reflects the accumulation during $24 \mathrm{~h}$ of focal $\mathrm{BBB}$ disruption. High binding affinity to plasma albumin and extracellular matrix proteins may explain the delayed initial CNS tissue accumulation but also the persisting presence of Gf within the brain parenchyma for up to 10 days $[9,18]$. The inflammatory cascade in EAE includes a plethora of effector mechanisms such as secretion of matrix metalloproteases and other digesting enzymes by immune cells [32] initiating tissue degradation. Proteins liberated during this process are likely to trap Gf once it passed the disrupted BBB [10]. The histological analysis applying fluorescent $\mathrm{Gf}$ likewise indicated a diffuse extracellular enrichment pattern. The clearance of Gf from the brain parenchyma after 14 days might be a result of a gradual loss of local molecule binding affinity as well as the general clearance and molecular turnover at lesion sites. We also noted a local uptake of Gf molecules by macrophages/microglia. However, we could not establish a correlation to repair mechanisms such as remyelination (data not shown).

Despite a delayed brain parenchymal enhancement Gf accumulated in CVO already $1 \mathrm{~h}$ after application. CVO are primarily exposed to Gf molecules due to the dense capillary network devoid of a tight BBB. Endothelial cells of the choroid plexus and other CVO express proteoglycans, fibronectin and other extracellular matrix proteins $[33,34]$, which may bind Gf. During CNS inflammation, such molecules are upregulated and mediate adhesion and subsequent transmigration of immune cells into the CNS $[33,35]$. Thus, adhesion to extracellular matrix proteins presented on CVO endothelium might explain both, Gf enhancement in CVO of healthy control mice and increased enhancement under inflammatory conditions.

\section{Conclusions}

In summary, in this first in vivo MRI investigation visualizing inflammatory alterations in the CVO, Gf enhancement correlated to disease severity as well as to time of onset, indicating an active participation of the CVO in CNS inflammation. Gf enhancement in CVO was a good discriminator between healthy and EAE animals. Our results suggest that Gf enhancement could serve as sensitive marker for different hallmarks of CNS inflammation, including molecular "arming", immune cell recruitment, $\mathrm{BBB}$ breakdown and tissue damage. However, extensive preclinical testing is needed before a clinical application of Gf. This is eligible since Gf could not only facilitate the paraclinical diagnosis and follow-up of neuroinflammatory diseases such as MS but may also help to elucidate basic principles of CNS immunity.

\section{Acknowledgements}

We thank Susanne Mueller and Susan Pikol for assistance with animal scanning, Nancy Nowakowksi for animal care, and Martina Paetzel for 

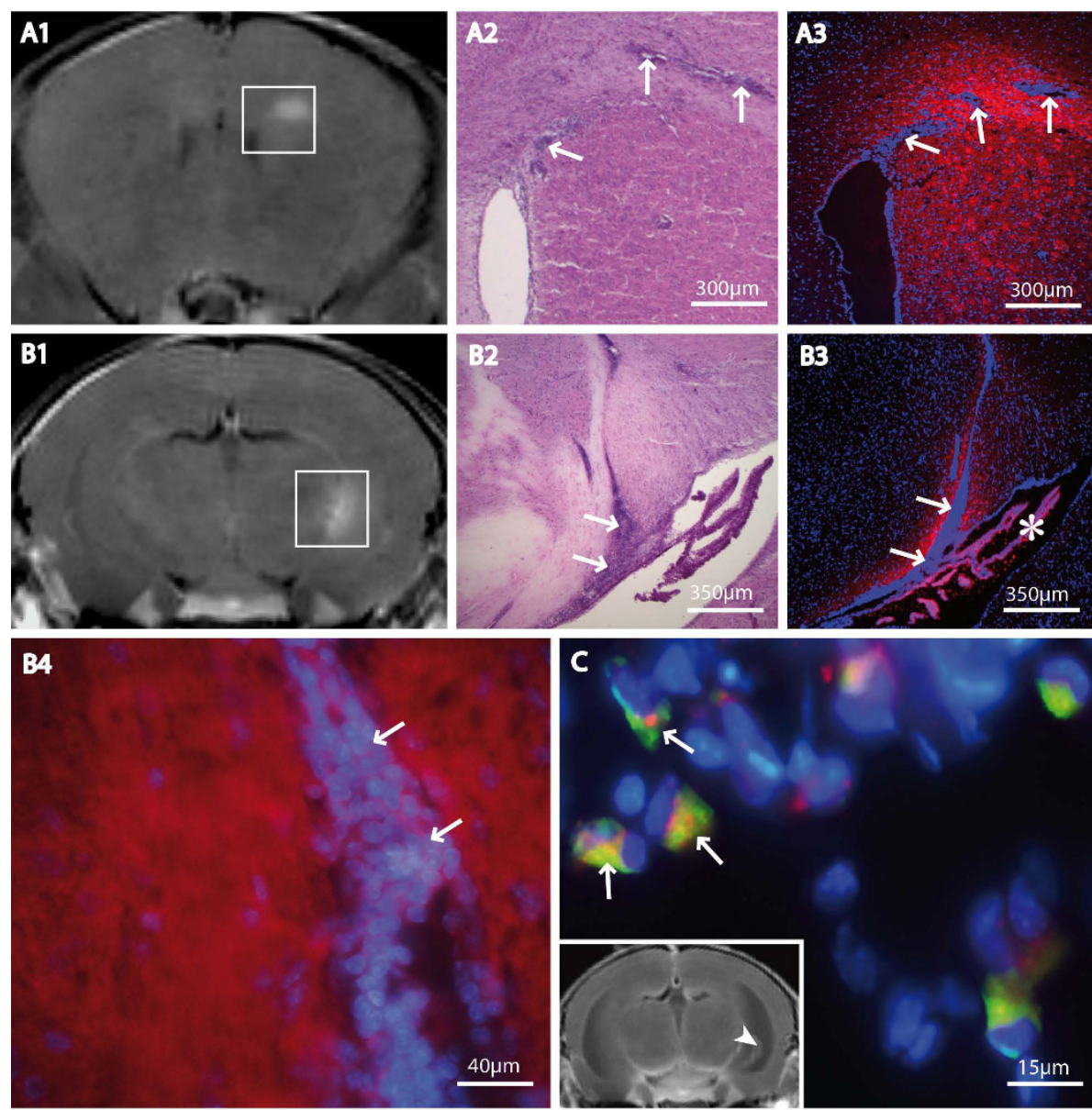

Figure 7 Histological correlations. The tissue distribution of fluorescence-labeled Gf was histologically assessed at two time points, $24 \mathrm{~h}$ (A and B) and $72 \mathrm{~h}(\mathrm{C})$ after intravenous Gf injection, and correlated to MRI. Coronal T1-weighted MR images $24 \mathrm{~h}$ after Gf injection are shown. Gfenhancing lesions are marked by squares (A1 and B1). Two exemplary lesions studied after $24 \mathrm{~h}$ are shown, one with a periventricular inflammatory focus close to the corpus callosum (A) and a second spreading from the hippocampal fimbria to the choroid plexus $\left(^{*}\right)(B)$. Corresponding H\&E stained (A2 and B2) and fluorescence microscopy (A3, B3, B4; red: Gf, blue: Hoechst 33258) slices depict typical inflammatory plaques, including cellular infiltrations (arrows), halo-like surrounded by Gf. A detail is given in higher magnification to point out the diffusely extracellular accumulation of Gf (B4). After 72 h, Gf uptake into macrophages/microglia was evident. In the choroid plexus, internalized Gf became visible in numerous IBA-1 positive cells (arrows in C; red: Gf; green: IBA-1; blue: Hoechst 33258). The MR image is shown to localize the detail (C: arrowhead).

correcting this manuscript as native English speaker. The authors declare no conflict of interest.

\section{Author details}

'Experimental and Clinical Research Center, Charité - University Medicine Berlin, Charitéplatz 1, 10117 Berlin, Germany. ${ }^{2}$ Department of Pediatrics, University Luebeck, Ratzeburger Allee 160, 23538 Luebeck, Germany. ${ }^{3}$ Institute of Neuroradiology, University Luebeck, Ratzeburger Allee 160, 23538 Luebeck, Germany.

\section{Authors' contributions}

EW carried out the animal experiments and histological procedures, performed the statistical analyses, participated in MR scanning and drafted the manuscript. CID participated in the design of the study and helped to draft the manuscript. RG helped with the histological stainings. JTW conceived and designed the study, performed MR scanning and drafted the manuscript. All authors read and approved the final manuscript.

\section{Competing interests}

The authors declare that they have no competing interests.

Received: 27 July 2010 Accepted: 18 October 2010

Published: 18 October 2010

\section{References}

1. Ransohoff RM, Kivisakk P, Kidd G: Three or more routes for leukocyte migration into the central nervous system. Nat Rev Immunol 2003, 3:569-581

2. Bechmann I, Galea I, Perry VH: What is the blood-brain barrier (not)? Trends Immunol 2007, 28:5-11.

3. Wittkowski W: Tanycytes and pituicytes: morphological and functional aspects of neuroglial interaction. Microsc Res Tech 1998, 41:29-42.

4. Lechan RM, Fekete C: Infundibular tanycytes as modulators of neuroendocrine function: hypothetical role in the regulation of the thyroid and gonadal axis. Acta Biomed 2007, 78(Suppl 1):84-98. 
5. Kivisakk P, Mahad DJ, Callahan MK, Trebst C, Tucky B, Wei T, Wu L, Baekkevold ES, Lassmann H, Staugaitis SM, et al: Human cerebrospinal fluid central memory CD4+ T cells: evidence for trafficking through choroid plexus and meninges via P-selectin. Proc Natl Acad Sci USA 2003, 100:8389-8394.

6. Engelhardt B, Wolburg-Buchholz K, Wolburg H: Involvement of the choroid plexus in central nervous system inflammation. Microsc Res Tech 2001, 52:112-129.

7. Schulz M, Engelhardt B: The circumventricular organs participate in the immunopathogenesis of experimental autoimmune encephalomyelitis. Cerebrospinal Fluid Res 2005, 2:8.

8. Wuerfel J, Tysiak E, Prozorovski T, Smyth M, Mueller S, Schnorr J, Taupitz M, Zipp F: Mouse model mimics multiple sclerosis in the clinico-radiological paradox. Eur J Neurosci 2007, 26:190-198.

9. Bendszus M, Ladewig G, Jestaedt L, Misselwitz B, Solymosi L, Toyka K, Stoll G: Gadofluorine M enhancement allows more sensitive detection of inflammatory CNS lesions than T2-w imaging: a quantitative MRI study. Brain 2008, 131:2341-2352.

10. Stoll G, Kleinschnitz C, Meuth SG, Braeuninger S, Ip CW, Wessig C, Nolte I, Bendszus M: Transient widespread blood-brain barrier alterations after cerebral photothrombosis as revealed by gadofluorine M-enhanced magnetic resonance imaging. J Cereb Blood Flow Metab 2008.

11. Misselwitz B, Platzek J, Weinmann HJ: Early MR lymphography with gadofluorine $M$ in rabbits. Radiology 2004, 231:682-688.

12. Sirol M, Itskovich W, Mani V, Aguinaldo JG, Fallon JT, Misselwitz B, Weinmann HJ, Fuster V, Toussaint JF, Fayad ZA: Lipid-rich atherosclerotic plaques detected by gadofluorine-enhanced in vivo magnetic resonance imaging. Circulation 2004, 109:2890-2896.

13. Bendszus M, Wessig C, Schutz A, Horn T, Kleinschnitz C, Sommer C, Misselwitz B, Stoll G: Assessment of nerve degeneration by gadofluorine M-enhanced magnetic resonance imaging. Ann Neurol 2005, 57:388-395.

14. Smorodchenko A, Wuerfel J, Pohl EE, Vogt J, Tysiak E, Glumm R, Hendrix S, Nitsch R, Zipp F, Infante-Duarte C: CNS-irrelevant T-cells enter the brain, cause blood-brain barrier disruption but no glial pathology. Eur $J$ Neurosci 2007, 26:1387-1398.

15. Aktas O, Smorodchenko A, Brocke S, Infante-Duarte C, Schulze Topphoff U, Vogt J, Prozorovski T, Meier S, Osmanova V, Pohl E, et al: Neuronal damage in autoimmune neuroinflammation mediated by the death ligand TRAIL. Neuron 2005, 46:421-432.

16. Aktas O, Waiczies S, Smorodchenko A, Dorr J, Seeger B, Prozorovski T, Sallach S, Endres M, Brocke S, Nitsch R, Zipp F: Treatment of relapsing paralysis in experimental encephalomyelitis by targeting Th1 cells through atorvastatin. J Exp Med 2003, 197:725-733.

17. Weinmann HJ, Brasch RC, Press WR, Wesbey GE: Characteristics of gadolinium-DTPA complex: a potential NMR contrast agent. AJR Am J Roentgenol 1984, 142:619-624.

18. Meding J, Urich M, Licha K, Reinhardt M, Misselwitz B, Fayad ZA, Weinmann $\mathrm{HJ}$ : Magnetic resonance imaging of atherosclerosis by targeting extracellular matrix deposition with Gadofluorine M. Contrast Media Mol Imaging 2007, 2:120-129.

19. Raatschen HJ, Swain R, Shames DM, Fu Y, Boyd Z, Zierhut ML, Wendland MF, Misselwitz B, Weinmann HJ, Wolf KJ, Brasch RC: MRI tumor characterization using Gd-GlyMe-DOTA-perfluorooctyl-mannoseconjugate (Gadofluorine M), a protein-avid contrast agent. Contrast Media Mol Imaging 2006, 1:113-120.

20. Wuerfel J, Bellmann-Strobl J, Brunecker $P$, Aktas $O$, McFarland $H$, Villringer $A$ Zipp F: Changes in cerebral perfusion precede plaque formation in multiple sclerosis: a longitudinal perfusion MRI study. Brain 2004, 127:111-119.

21. Engelhardt B, Ransohoff RM: The ins and outs of T-lymphocyte trafficking to the CNS: anatomical sites and molecular mechanisms. Trends Immunol 2005, 26:485-495.

22. Galea I, Bechmann I, Perry VH: What is immune privilege (not)? Trends Immunol 2007, 28:12-18.

23. Carrithers MD, Visintin I, Viret C, Janeway CS Jr: Role of genetic background in $\mathrm{P}$ selectin-dependent immune surveillance of the central nervous system. J Neuroimmunol 2002, 129:51-57.

24. Serafini B, Columba-Cabezas S, Di Rosa F, Aloisi F: Intracerebral recruitment and maturation of dendritic cells in the onset and progression of experimental autoimmune encephalomyelitis. Am J Pathol 2000, 157:1991-2002.
25. Brown DA, Sawchenko PE: Time course and distribution of inflammatory and neurodegenerative events suggest structural bases for the pathogenesis of experimental autoimmune encephalomyelitis. J Comp Neurol 2007, 502:236-260.

26. Lindsley MD, Patick AK, Prayoonwiwat N, Rodriguez M: Coexpression of class I major histocompatibility antigen and viral RNA in central nervous system of mice infected with Theiler's virus: a model for multiple sclerosis. Mayo Clin Proc 1992, 67:829-838.

27. Vellinga MM, Oude Engberink RD, Seewann A, Pouwels PJ, Wattjes MP, van der Pol SM, Pering C, Polman CH, de Vries HE, Geurts JJ, Barkhof F: Pluriformity of inflammation in multiple sclerosis shown by ultra-small iron oxide particle enhancement. Brain 2008, 131:800-807.

28. Barkhausen J, Ebert W, Heyer C, Debatin JF, Weinmann HJ: Detection of atherosclerotic plaque with Gadofluorine-enhanced magnetic resonance imaging. Circulation 2003, 108:605-609.

29. Henning TD, Saborowski O, Golovko D, Boddington S, Bauer JS, Fu Y, Meier R, Pietsch H, Sennino B, McDonald DM, Daldrup-Link HE: Cell labeling with the positive MR contrast agent Gadofluorine M. Eur Radiol 2007, 17:1226-1234.

30. Stoll G, Wessig C, Gold R, Bendszus M: Assessment of lesion evolution in experimental autoimmune neuritis by gadofluorine M-enhanced MR neurography. Exp Neurol 2006, 197:150-156.

31. Schmidt S, Vieweger A, Obst M, Mueller S, Gross V, Gutberlet M, Steinbrink J, Taubert S, Misselwitz B, Luedemann L, Spuler S: Dysferlindeficient muscular dystrophy: gadofluorine $M$ suitability at MR imaging in a mouse model. Radiology 2009, 250:87-94.

32. Gold R, Linington C, Lassmann H: Understanding pathogenesis and therapy of multiple sclerosis via animal models: 70 years of merits and culprits in experimental autoimmune encephalomyelitis research. Brain 2006, 129:1953-1971.

33. Silva AA, Roffe $E$, Lannes-Vieira J: Expression of extracellular matrix components and their receptors in the central nervous system during experimental Toxoplasma gondii and Trypanosoma cruzi infection. Braz $J$ Med Biol Res 1999, 32:593-600.

34. Taguchi T, Ohtsuka A, Murakami T: Light and electron microscopic detection of anionic sites in the rat choroid plexus. Arch Histol Cytol 1998, 61:243-252.

35. Venstrom KA, Reichardt LF: Extracellular matrix. 2: Role of extracellular matrix molecules and their receptors in the nervous system. Faseb J 1993, 7:996-1003.

doi:10.1186/1742-2094-7-70

Cite this article as: Wuerfel et al:: Gadofluorine M-enhanced MRI shows involvement of circumventricular organs in neuroinflammation. Journal of Neuroinflammation 2010 7:70.

\section{Submit your next manuscript to BioMed Central and take full advantage of:}

- Convenient online submission

- Thorough peer review

- No space constraints or color figure charges

- Immediate publication on acceptance

- Inclusion in PubMed, CAS, Scopus and Google Scholar

- Research which is freely available for redistribution

Submit your manuscript at www.biomedcentral.com/submit
C Biomed Central 